\title{
High-speed Rayleigh-Raman measurements with subframe burst gating
}

\author{
Yedhu Krishna, hao Tang, Ayman M. Elbaz and Gaetano Magnotti*
}

Clean Combustion Research Center, King Abdullah University of Science and Technology (KAUST), Thuwal 23955-6900, Saudi Arabia.

*Corresponding author: Gaetano.magnotti@kaust.edu.sa

Received XX Month XXXX; revised XX Month, XXXX; accepted XX Month XXXX; posted XX Month XXXX (Doc. ID XXXXX); published XX Month XXXX

A $10 \mathrm{kHz}$ one-dimensional Rayleigh- $\mathrm{CH}_{4}$ Raman instrument capable of achieving highly precise measurement of temperature and methane mole fraction is demonstrated. The system uses a pulse burst laser as the light source and back-illuminated electron-multiplied CCD cameras as the detectors. The cameras are operated in subframe burst gating mode, to combine high sampling rate, with low-noise, slow-readout. The improved precision of this configuration is demonstrated by measuring temperature and methane mole fraction in ambient temperature gas mixtures and in a non-premixed inverse diffusion flame.

http://dx.doi.org/10.1364/OL.99.099999

One-dimensional Rayleigh-Raman scattering is a powerful diagnostic to measure temperature and major species concentration in non-sooting flames with high accuracy and precision, but with sampling rates typically limited to 5 or $10 \mathrm{~Hz}$ [1]. The study of combustion dynamics events such as quenching, extinction, and thermo-acoustics instabilities, would greatly benefit from extending the Rayleigh/ Raman diagnostics to kHz rates, while maintaining comparable accuracy and precision. Raman scattering in combustion requires laser pulse energies of the order of $1 \mathrm{~J}$, which is 2 orders of magnitude higher than what is provided by conventional high-speed lasers. Only thanks to the recent development of high-energy pulse-burst lasers [2, 3] that can operate at several $\mathrm{kHz}$ and deliver pulses with hundreds of millijoules, Rayleigh and Raman scattering in combustion have been extended to $\mathrm{kHz}$ rates $[4,5]$.

The high precision and accuracy of low-repetition-rate Raman systems are enabled by using low-noise CCD cameras, which are generally limited to an acquisition rate of $5 \mathrm{~Hz}$. Previous pulse-burst Rayleigh-Raman scattering instruments employed high-speed CMOS cameras as detectors due to their high acquisition rate $[4,5]$. High-speed CMOS cameras have inferior noise performance, lower dynamic range, and poorer quantum efficiency than backilluminated CCD cameras, resulting in lower signal-to-noise ratio (SNR) and instrument precision. In particular for pulse-burst Raman spectroscopy the scattered signal is weak, and a high-speed intensifier is needed, to boost the signal on the CMOS camera while suppressing flame luminosity. The use of high-speed intensifier provides a fast gating that suppresses flame luminosity, but results in poorer precision and loss of spatial resolution. Ajrouche et al. [6] demonstrated that electron multiplying CCD (EMCCD) cameras can provide a higher signal to noise ratio than ICCD camera for weak Raman signal, and are better detectors to extend the detection limit beyond the readout noise limit. An external shutter that consists of a Pockels cell and two crossed polarizers can provide exposure times of a few hundred nanoseconds [7]. This approach can be extended to $10 \mathrm{kHz}$ rate using off-the-shelflongitudinal Pockels cell.

In this paper, we introduce the first pulse burst RayleighRaman instrument featuring low-noise, back-illuminated EMCCD cameras operated in subframe burst gating mode $[8,9]$, to achieve repetition rates of $10 \mathrm{kHz}$ without compromising the measurement precision. The system is demonstrated by conducting simultaneous temperature and methane mole fraction measurements in ambient-temperature gas mixtures and in a non-premixed turbulent inverse diffusion flame. For all test-cases considered, the achieved instrument precision is higher than the previously reported values. The subframe burst gating allows combining $\mathrm{kHz}$ rate acquisition to the low-noise performance of CCD cameras. In this mode of operation, only the bottom $\mathrm{N}$ pixel rows of the camera chip are exposed and the remaining of the chip is masked. After each laser pulse, the accumulated photocharge is sequentially shifted upward by $\mathrm{N}$ rows to the masked region $[8,9]$. This process continues until the entire chip is filled. Low-noise, slow-readout through the serial register is then possible, because it does not interfere with the high-speed sampling. A drawback is that the number of recorded images is limited by the camera sensor size. Pulse-burst lasers provide high repetition rate within a burst, but typical burst rates are $<0.1 \mathrm{~Hz}$, allowing sufficient time for a slow readout between bursts. Subframe burst gating is therefore an ideal, although unexplored, match for pulse burst laser diagnostics.

Fig. 1 shows the schematic diagram of the optical setup. The different parts of the system are described below. The second harmonic output of a Spectral Energies "Quasimodo" pulse burst laser operated at $10 \mathrm{kHz}$ with $350 \mathrm{~mJ} /$ pulse provides the Rayleigh/ Raman excitation source. The maximum burst duration is $10 \mathrm{~ms}$, resulting in a maximum of 100 consecutive pulses. The laser beam is focused at the measurement volume using a $500 \mathrm{~mm}$ lens. The 
estimated beam spot size is $\sim 100 \mu \mathrm{m}$. Optical breakdown is avoided by operating the laser in "giant pulse" mode, temporally stretching the laser pulse to a full-width at half-maximum of $100 \mathrm{~ns}$. After passing the probe region, the laser beam is re-collimated and directed to a high-speed energy meter (Coherent J-50MT-10KHZ), for shot-to-shot energy correction.

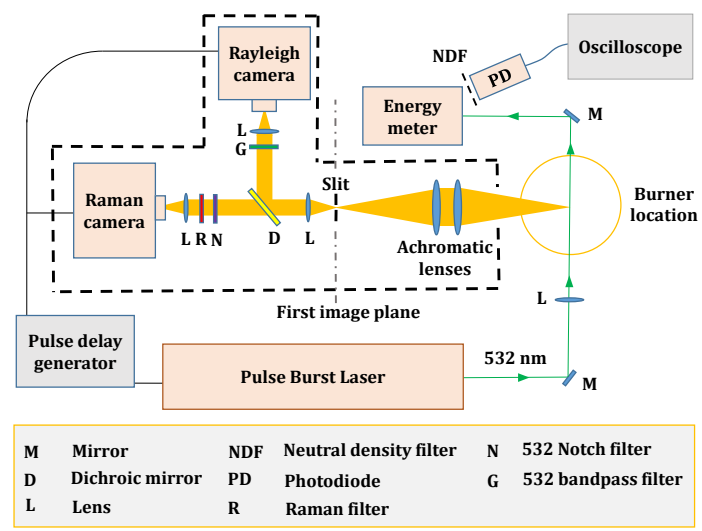

Fig. 1. Schematic diagram of the high-speed Rayleigh-Raman set up.

A pair of air-spaced, three-element achromatic lenses with a diameter of $100 \mathrm{~mm}$, and focal lengths of $400 \mathrm{~mm}$ and $300 \mathrm{~mm}$, collects and relays the Rayleigh/Raman signal to the "first image plane". The lens diameter and focal distance were chosen to fit in the arm of the high-pressure combustion duct at KAUST [10]. The light is then collected by a multi-element Nikon lens of focal length $85 \mathrm{~mm}$ and an f-number of 1.8, and collimated to a dichroic mirror with a cut off wavelength at $550 \mathrm{~nm}$. The dichroic mirror splits the collected signal into two: the Rayleigh signal at $532 \mathrm{~nm}$ is reflected at $90^{\circ}$ to the Rayleigh camera and the Raman signal around $630 \mathrm{~nm}$ is transmitted to the Raman camera, as shown in Fig. 1. The cameras are two back-illuminated EMCCD cameras (Princeton Instruments ProEM-HS) synchronized with the laser burst via a delay generator. A multi-element Nikon lens of $50 \mathrm{~mm}$ focal length and f-number 1.8 focuses the image on the camera chip. The Raman camera has two filters in front of its collection lens: a $532 \mathrm{~nm}$ notch filter of optical density 6 to block any remaining $532 \mathrm{~nm}$ light leaking through the dichroic and a bandpass filter with a center wavelength of $635 \mathrm{~nm}$ and a bandwidth of $18 \mathrm{~nm}$ to pass only the Raman signal of $\mathrm{CH}$ stretch region of the $\mathrm{CH}_{4}$ molecule. A $532 \mathrm{~nm}$ bandpass filter with a bandwidth of $10 \mathrm{~nm}$ and optical density 4 is placed in front of the collection lens of the Rayleigh camera to cut stray light outside the $532 \mathrm{~nm}$ region. Both cameras are placed on a translation stage for fine adjustment of focus. Spatial registration of the two cameras is performed using a pinhole and a target consisting of a dot matrix with $125 \mu \mathrm{m}$ spacing. This step is important since the Raman images at a given spatial location have to be used in conjunction with the Rayleigh images at the corresponding location to obtain accurate and meaningful results. The total magnification of the collection system is $\sim 0.79$ on both cameras. The entire collection optics is covered with black-out materials to avoid stray light.

For operation in the subframe burst gating mode, generally a mask is applied directly to the chip $[8,9,11]$, but this strategy prevents from using the camera in the full frame mode in other experiments. To maintain this capability, in our configuration the masking of the camera is achieved by placing a slit at the first image plane as illustrated in Fig. 1. This allows higher flexibility because the exposed window can be changed easily by varying the opening of the slit. In our experiment, a window height of $25 \mathrm{X} 1024$ pixels is used. With a resolution of 1024 X 1024 and an equally big frame transfer area, this window size allows a total of 81 images to be acquired during a single burst. The window height of 25 pixels in the vertical direction on the camera, corresponding to a height of $\sim 410$ $\mu \mathrm{m}$ in the target region, makes sure that the signal is not clipped by the modest beam steering observed during a burst. A major advantage of CCD cameras compared to CMOS cameras is the possibility of hardware binning for superior noise reduction compared to software binning. Hardware binning over the 25-pixel height of the acquisition window is performed to boost the $\mathrm{CH}_{4}$ Raman signal. The signal on the Rayleigh camera is sufficiently strong and no hardware binning is required. This results in a larger dynamic range, and also allows us to verify that the Rayleigh signal was completely within the slit opening, despite beam-steering and changes in the focusing during the burst. Slow readout (1 MHz) CCD mode provides the best signal-to-noise performance when sufficient light is available, and is used for Rayleigh measurements of temperature, and for measurements of methane mole fraction in non-reacting jets. The Raman camera is operated in the EMCCD mode with a gain of 80 for measurements in the flame, sacrificing instrument precision for lower detection limits.

A non-premixed turbulent inverse diffusion flame is used to demonstrate the instrument in a reactive environment. The burner consists of two concentric stainless steel tubes - the central tube (ID $10.7 \mathrm{~mm}$, OD $12.6 \mathrm{~mm}$ ) supplies air and the outer tube (ID $15 \mathrm{~mm}$, OD $19 \mathrm{~mm}$ ) supplies fuel. This is a modified version (larger tube diameters) of the flame investigated in [12]. A tailored mixture of hydrogen, methane and nitrogen in the ratio $33.2 \%, 22.1 \%$ and $44.7 \%$ respectively (by volume) is chosen as fuel to provide an approximately constant effective Rayleigh cross section across the flame [13]. The fuel flow rate is 6 standard liters per minute (SLPM) and the central air flow rate is 85.7 SLPM corresponding to a Reynolds number of 12000 . A coflow of air at 296 SLPM provides a laminar shroud surrounding the flame. Measurements are conducted at three different heights $-5 \mathrm{~mm}, 10 \mathrm{~mm}$ and $20 \mathrm{~mm}$ from the fuel tube exit.

The signal collected on the Rayleigh camera is software-binned in the vertical and horizontal direction (bin size: 25 and 6, respectively) to improve the SNR and to obtain a probe volume size of $100 \mu \mathrm{m}$. The average of 100 images taken in presence of the flame, and with the laser blocked, is binned and subtracted to each Rayleigh image to account for the camera offset, residual ambient illumination and flame luminosity. The exposure time is $100 \mu \mathrm{s}$, and the corresponding flame luminosity signal is negligible compared to the Rayleigh scattering signal. The Rayleigh signal is then scaled by the instantaneous energy meter reading to account for laser energy fluctuations. Measurements in dry air at a known temperature provide the needed calibration curve $R a y_{\text {air }}$. If $T_{\text {air }}$ is the temperature in dry air measured with a thermocouple, Ray is the measured Rayleigh signal, $\sigma_{n}$ the normalized Rayleigh cross section defined as the ratio of the Rayleigh cross section of the sample mixture and of air, then the temperature is given by:

$$
T=T_{\text {air }} \frac{\text { Ray }_{\text {air }}}{\text { Ray }} \sigma_{n}
$$

For the tailored mixture, the Rayleigh cross section across the flame can be approximated to be a constant $\left(\sigma_{n} \sim 1\right)$, decoupling 
temperature and species measurements. The same binning and background correction is applied to the image collected on the Raman camera to obtain the Raman signal ( $R a m)$. A 1D calibration curve $\mathrm{Ram}_{\mathrm{CH} 4}$ is obtained from measurements in a uniform flow of methane. The temperature in the methane flow $T_{C H 4}$ is measured using a thermocouple. Then the mole fraction of methane is:

$$
X_{C H 4}=\frac{T}{T_{C H 4}} \frac{\operatorname{Ram}}{\operatorname{Ram}_{C H 4}} C(T)
$$

where $T$ is the temperature measured from Rayleigh scattering and $c(T)$ is an empirical polynomial that takes into account the change in the integrated intensity of the density-weighted methane Raman spectrum as a function of temperature [14].

The flame luminosity at its peak is $1 \%$ of the Raman signal in the unburnt tailored mixture, therefore it cannot be neglected. Since the Raman signal is inversely proportional to temperature, the effect of flame luminosity on the mole fraction is approximately equal to $0.0022 \frac{T}{T_{\text {cold }}}$. The flame luminosity is recorded at each location, and subtracted from the Raman signal. Luminosity fluctuations are less than $\pm 10 \%$ for the flame considered, leading to \pm 0.0012 error in the single-shot mole fraction, which is much smaller than other sources of error.
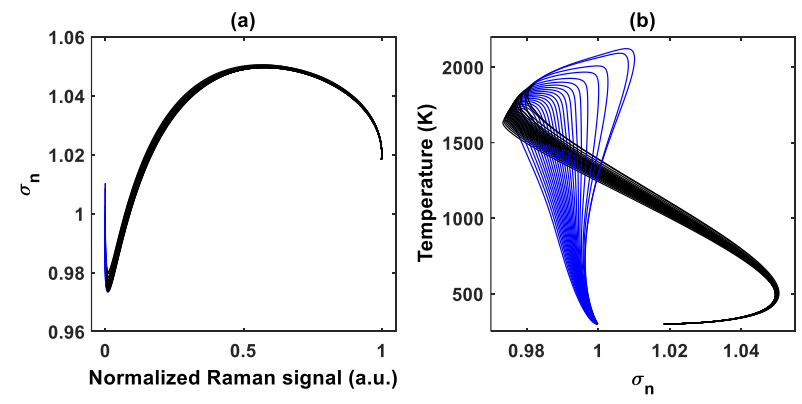

Fig. 2. Variation of (a) $\sigma_{\mathrm{n}}$ vs normalized Raman signal, (b) temperature vs $\sigma_{n}$, for various strain rates. The blue curves indicates the region where the normalized Raman signal is less than $2 \%$.

The use of a tailored mixture decouples the temperature measurements from the species measurements, so that the precision of the proposed high-speed Raman and Rayleigh can be assessed independently. Simultaneous Rayleigh/ $\mathrm{CH}_{4}$ Raman at 10 $\mathrm{Hz}$ has been used in the past to obtain temperature measurements in non-premixed jet flames [15]. The sample composition, necessary to compute the Rayleigh cross section, was estimated by combining the fuel number density measurements to a flamelet model. In this work we use the same approach to improve the accuracy of the temperature/species measurements obtained using the tailored mixture. Laminar flame calculations of counterflow diffusion flames for different strain rates are shown in Fig. 2. Fig. 2(a) shows the Rayleigh cross section as a function of the Raman signal normalized by their respective values in the cold tailored mixture. For a given normalized Raman signal, the Rayleigh cross section varies only within $\pm 0.2 \%$ for different strain rates, providing an improved estimate of the Rayleigh cross section. When the normalized Raman signal is less than $2 \%$, the Rayleigh cross section is estimated from the temperature, based on Fig.2(b) (blue region). The average peak temperature at the probed location is used to select the strain rate of the $1 \mathrm{D}$ counterflow flame calculations used for the correction. For the tailored mixture, the effect of this correction is small: $\sim-40$ Kat most in temperature and $\sim .007$ in CH4 mole fraction, but it would be critical for non-tailored fuels where the Rayleigh cross section doubles going from air to fuel.

Preliminary measurements in ambient air were performed to directly compare the precision of $1 \mathrm{D}, 10 \mathrm{kHz}$ Rayleigh measurements using the EMCCD camera (Princeton ProEM-HS) operated in the subframe burst gating mode and a high-speed CMOS camera (LaVision Imager pro HS). Energy fluctuations were monitored using a photodiode (Thorlabs DET025A). The energy of the laser was reduced to $1 / 6^{\text {th }}$ of the available laser energy to reproduce the signal level expected in a flame at $1800 \mathrm{~K}$. The signal was software binned to provide a spatial resolution of $78 \mu \mathrm{m}$ and $77 \mu \mathrm{m}$ on the EMCCD and CMOS cameras respectively. The coefficient of variation (COV), defined as the standard deviation over a single laser burst expressed as the percentage of the mean, is used here as an indication of the instrument precision. For equal conditions, the EMCCD camera achieved a COV of $<1 \%$ that is half of what was achieved with the employed mid-range high-speed CMOS camera (2\%). Note that this is not a general result, but highly specific to the experimental set-up used, and to the intensity of the collected signal. CMOS performances approach those of CCD cameras as the signal amplitude increases. As an example Hoffmeister [4] reported a COV of $1 \%$ for $10 \mathrm{kHz}$ measurements in air and of $>2 \%$ for measurements in the product region of a laminar flat flame at a temperature of $\sim 1100 \mathrm{~K}$ due to the lower signal level. By replacing the photodiode with a pyroelectric high-speed energy meter, monitoring of the laser energy fluctuations improved, and a COV of $0.45 \%$ was obtained when using the EMCCD camera. This is very close to the $0.4 \%$ reported in state-of-the-art $5 \mathrm{~Hz}$ Raman/Rayleigh system [14]. To our knowledge, this is the lowest COV reported for Rayleigh measurements in air at $10 \mathrm{kHz}$.

The Raman signal is significantly weaker than the Rayleigh signal, and measurements with the CMOS camera were too noisy to process even in pure jets of methane at ambient temperature. Previously reported high-speed Raman measurements were taken using high-speed IRO in front of the CMOS camera [4, 5]. It has been extensively reported that the SNR from an ICCD camera is better than for an EMCCD camera only at very low-light conditions (1-10 photons/pixel) [6, 16]. For measurements of CO in flames at $10 \mathrm{~Hz}$, Ajrouche [6] reported a COV of $9.2 \%$ using a back-illuminated CCD camera, $11.2 \%$ using a back-illuminated EMCCD, and $17.5 \%$ when using an ICCD. For measurements in a uniform flow of methane, we recorded a COV of $0.6 \%$ when the EMCCD camera is operated in low-noise CCD mode and 1.2\% when operated in EMCCD mode, confirming that the EM-mode does not improve the precision other than for very weak signal. For Raman measurements in flames, notwithstanding the lower precision, the EMCCD mode was preferred to ensure sufficient Raman signal within the flame where the methane concentration is very low. Measurements of methane in a tailored mixture containing $22.1 \%$ of methane have a COV of $1.8 \%$ using the EMCCD mode with a gain of 80 . As a reference, Hoffmeister [4] reported a 3.3\% COV for nitrogen in air when using the IRO+CMOS combination, higher laser energies and more efficient collection. Fig. 3 shows the mean and standard deviation of the temperature and mole fraction measurements at three different heights in the flame. The flame is symmetric and the measurements on only one side of the flame are shown. Scatter plots of $\mathrm{CH}_{4}$ mole fraction versus temperature are also shown in this figure. 10 to 15 burst measurements were taken at each location, depending on the 
flow condition to obtain statistically significant measurements of mean and standard deviation.
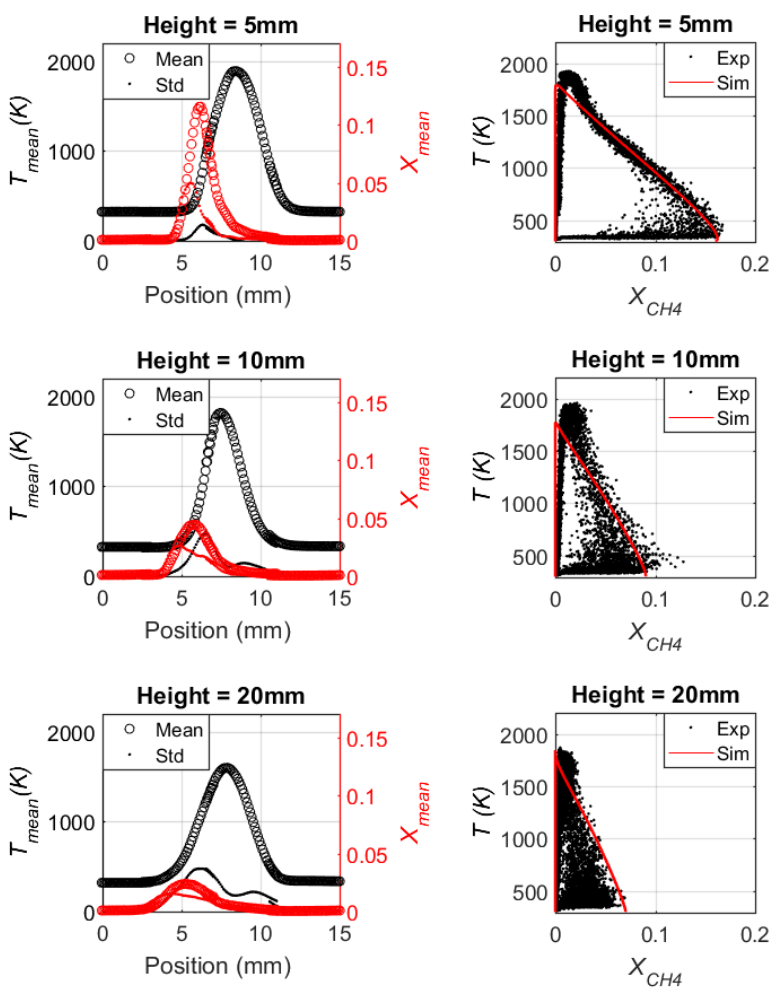

Fig. 3. (a) The mean and standard deviation of temperature and methane mole fraction, and (b) the scatter plot between methane mole fraction and temperature at different heights for the flame.

At $5 \mathrm{~mm}$ height from the burner exit, mixing between the center jet and the fuel annular stream has the effect of partially premixing the fuel mixture, lowering the methane concentration. A laminar flame is formed at the interface between the partially premixed fuel jet and the slow-moving co-flow of air. This is confirmed by the good agreement between the scatter plot with the numerical results of a laminar opposed non-premixed flame with a composition of $16 \%$ methane to match the measured peak value of mole fraction. The methane standard deviation profiles peaks at the mixing layer between the turbulent air central jet and the tailored fuel jet (5.5 $\mathrm{mm}$ away from the axis). The peak in the temperature standard deviation indicates the mixing between the preheat zone of the laminar flame and the turbulent jet. In correspondence of the peak temperature $(>1800 \mathrm{~K}$ ) the $\mathrm{COV}$ is $0.67 \%$, further confirming the higher temperature precision offered by this instrument. As a reference, the Sandia $10 \mathrm{~Hz}$ Rayleigh-Raman instrument achieved $0.8 \%$ at $2100 \mathrm{~K}$ [14], where the pulse-burst $10 \mathrm{kHz}$ system described by Hoffmeister [4] achieved $\sim 2 \%$ at $1300 \mathrm{~K}$. The highprecision at elevated temperature further confirms that sub-frame burst gate readout can extend the precision of $10 \mathrm{~Hz}$ systems to pulse-burst diagnostics. As we move downstream to $10 \mathrm{~mm}$ and 20 $\mathrm{mm}$ heights, the flow becomes more turbulent and increased mixing of the fuel with the central air jet reduces the $\mathrm{CH}_{4}$ mole fraction to $<0.05$ and the standard deviation in mole fraction increases to $>50 \%$ of the mean. The temperature profile is significantly broadened, and the peak temperature becomes lower.
Scatter plot at the downstream conditions shows data points completely filling the region enveloped by the laminar flame calculations, reflecting the large variation in the composition of the partially premixed jet interacting with the co-flow air. Changes in composition on the fuel side, prevent the use of this approach in inverse diffusion flames with non-tailored fuel mixtures.

In summary, for the first time, we combined sub-frame burst gate readout to pulse-burst Rayleigh- $\mathrm{CH}_{4}$ Raman, to achieve high-speed data acquisition with no loss of precision. The measured temperature precision of $0.45 \%$ in air and $0.67 \%$ at flame temperature $1800 \mathrm{~K}$ are the best precision reported in the literature for $10 \mathrm{kHz}$ Rayleigh measurements. Raman measurements of methane in cold gases had a precision of $0.8 \%$ when collected using CCD mode and $1.2 \%$ in EMCCD mode. Measurements have been demonstrated in weakly luminous turbulent flames, and profiles of temperature and methane mole fraction have been presented.

Acknowledgment. This research was supported by funding from King Abdullah University of Science and Technology (KAUST).

\section{References}

1. R. S. Barlow, Proceedings of the Combustion Institute 31, 49-75 (2007).

2. F. Fuest, M. J. Papageorge, W. R. Lempert, and J. A. Sutton, Opt. Lett.

37, 3231-3233 (2012).

3. M. N. Slipchenko, J. D. Miller, S. Roy, J. R. Gord, S. A. Danczyk, and T. R. Meyer, Opt. Lett. 37, 1346-1348 (2012).

4. K. N. Gabet Hoffmeister, F. Fuest, and J. A. Sutton ,AIAA 2015-0531 (2015).

5. N. Jiang, P. S. Hsu, J. G. Mance, Y. Wu, M. Gragston, Z. Zhang, J. D. Miller, J. R. Gord, and S. Roy, Opt. Lett. 42, 3678-3681 (2017).

6. H. Ajrouche;, A. Lo;, A. P. Vervisch;, and A. Cessou, 18th International Symposium on the Application of Laser and Imaging Techniques to Fluid Mechanics (2016).

7. H. Ajrouche, A. Lo, P. Vervisch, and A. Cessou, Measurement Science and Technology 26, 075501 (2015)

8. M. B. Sinclair, D. M. Haaland, J. A. Timlin, and H. D. T. Jones, Appl. Opt. 45, 6283-6291 (2006).

9. J. Kojima, D. Fischer, and Q.-V. Nguyen, Opt. Lett. 35, 1323-1325 (2010).

10. W. R. Boyette, A. M. Elbaz, T. F. Guiberti, and W. L. Roberts, Experimental Thermal and Fluid Science 105, 332-341 (2019).

11. C. N. Dennis, A. Satija, and R. P. Lucht, Journal of Raman Spectroscopy 47, 177-188 (2016).

12. A. M. Elbaz, and W. L. Roberts, Combustion Science and Technology 186, 1249-1272 (2014).

13. V. Bergmann, W. Meier, D. Wolff, and W. Stricker, Applied Physics B 66, 489-502 (1998).

14. G. Magnotti, and R. S. Barlow, Combustion and Flame 162, 100-114 (2015).

15. S. P. Kearney, R. W. Schefer, S. J. Beresh, and T. W. Grasser, Appl. Opt. 44, 1548-1558 (2005).

16. D. Dussault, and P. Hoess, Proc. SPIE 556, 195 (2004).

\section{References with titles}

1. R. S. Barlow, "Laser diagnostics and their interplay with computations to understand turbulent combustion," Proceedings of the Combustion Institute 31, 49-75 (2007).

2. F. Fuest, M. J. Papageorge, W. R. Lempert, and J. A. Sutton, "Ultrahigh laser pulse energy and power generation at $10 \mathrm{kHz}$," Opt. Lett. 37, 32313233 (2012).

3. M. N. Slipchenko, J. D. Miller, S. Roy, J. R. Gord, S. A. Danczyk, and T. R. Meyer, "Quasi-continuous burst-mode laser for high-speed planar imaging," Opt. Lett. 37, 1346-1348 (2012). 
4. K. N. Gabet Hoffmeister, F. Fuest, and J. A. Sutton, "High-Speed 1D Raman/Rayleigh Scattering Imaging in Turbulent H2/N2 Flames," in 53rd AIAA Aerospace Sciences Meeting, AIAA SciTech Forum (American Institute of Aeronautics and Astronautics, 2015),

5. N. Jiang, P. S. Hsu, J. G. Mance, Y. Wu, M. Gragston, Z. Zhang, J. D.

Miller, J. R. Gord, and S. Roy, "High-speed 2D Raman imaging at elevated pressures," Opt. Lett. 42, 3678-3681 (2017).

6. H. Ajrouche, A. Lo;, A. P. Vervisch;, and A. Cessou, "Detector Assessment for 1 D Single - Shot Spontaneous Raman Scattering for Temperature and Multi - Species Measurements in flames

" in 18th International Symposium on the Application of Laser and Imaging Techniques to Fluid Mechanics, 2016),

7. H. Ajrouche, A. Lo, P. Vervisch, and A. Cessou, "Assessment of a fast electro-optical shutter for 1D spontaneous Raman scattering in flames," Measurement Science and Technology 26, 075501 (2015).

8. M. B. Sinclair, D. M. Haaland, J. A. Timlin, and H. D. T. Jones, "Hyperspectral confocal microscope," Appl. Opt. 45, 6283-6291 (2006).

9. J. Kojima, D. Fischer, and Q.-V. Nguyen, "Subframe burst gating for Raman spectroscopy in combustion," Opt. Lett. 35, 1323-1325 (2010).

10. W. R. Boyette, A. M. Elbaz, T. F. Guiberti, and W. L. Roberts,

"Experimental investigation of the near field in sooting turbulent nonpremixed flames at elevated pressures," Experimental Thermal and Fluid Science 105, 332-341 (2019).

11. C. N. Dennis, A. Satija, and R. P. Lucht, "High dynamic range thermometry at $5 \mathrm{kHz}$ in hydrogen-air diffusion flame using chirped-probepulse femtosecond coherent anti-stokes Raman scattering," Journal of Raman Spectroscopy 47, 177-188 (2016).

12. A. M. Elbaz and W. L. Roberts, "Experimental Characterization of Methane Inverse Diffusion Flame," Combustion Science and Technology 186, 1249-1272 (2014).

13. V. Bergmann, W. Meier, D. Wolff, and W. Stricker, "Application of spontaneous Raman and Rayleigh scattering and 2D LIF for the characterization of a turbulent $\mathrm{CH} 4 / \mathrm{H} 2 / \mathrm{N} 2$ jet diffusion flame," Applied Physics B 66, 489-502 (1998).

14. G. Magnotti and R. S. Barlow, "Effects of high shear on the structure and thickness of turbulent premixed methane/air flames stabilized on a bluff-body burner," Combustion and Flame 162, 100-114 (2015).

15. S. P. Kearney, R. W. Schefer, S. J. Beresh, and T. W. Grasser,

"Temperature imaging in nonpremixed flames by joint filtered Rayleigh and Raman scattering," Appl. Opt. 44, 1548-1558 (2005).

16. D. Dussault and P. Hoess, "Noise performance comparison of ICCD with CCD and EMCCD cameras," in Optical Science and Technology, the SPIE 49th Annual Meeting, (SPIE, 2004), 10. 